\title{
EL DESARROLLO DE UN CUESTIONARIO PARA PADRES PARA LA MEDICIÓN DE LA RESPONSIVIDAD SENSORIAL EN NIÑOS CON DIAGNÓSTICO DE AUTISMO (CMRS)
}

\author{
THE DEVELOPMENT OF A PARENTAL QUESTIONNAIRE TO MEASURE SENSORY \\ RESPONSIVITY IN CHILDREN WITH A DIAGNOSIS OF AUTISM (QMSR)
}

\section{Gustavo Reinoso ${ }^{1}$}

\begin{abstract}
Resumen
Este estudio presenta el desarrollo de un Cuestionario para Padres para la Medición de la Responsividad Sensorial en Niños con Diagnóstico de Autismo (CMRS). Se llevaron a cabo una revisión sistemática de la literatura internacional en el período $2000-2015$ y tres estudios Delphi. Los estudios Delphi examinaron prospectivamente a ochenta y siete $(N=87)$, setenta y tres $(N=73)$ y sesenta $(N=$ 60) Terapeutas Ocupacionales certificados en Integración Sensorial en Argentina. Los profesionales clasificaron 62 ítems en los rótulos de "mantener", "modificar" o "eliminar" los ítems del CMRS y proporcionaron cambios en su estructura y redacción. Adicionalmente, determinaron su inclusión en cuatro categorías: "hipo-responsividad", "hiper-responsividad", "planificación motriz" y "social" y dentro de seis escalas de responsividad sensorial: "visual", "auditiva", "vestibular", "gusto/olfato", "propioceptiva" y "táctil". Se presenta la versión final del CMRS y la retroalimentación profesional en todos sus ítems, categorías y escalas así como también sugerencias para estudios futuros que podrían proporcionar información adicional para su aplicación clínica y en investigación.
\end{abstract}

\section{Palabras Clave:}

Autismo, responsividad sensorial, cuestionarios, instrumentos de medición.

\begin{abstract}
This study presents the development of a Parental Questionnaire for the Measurement of Sensory Responsivity in Children with a Diagnosis of Autism (QMSR). A systematic review of the international literature in the 2000-2015 period and three Delphi studies were conducted. The three Dephi studies prospectively examined eighty-seven $(N=87)$, seventy-three $(N=73)$ and sixty $(N=60)$ Argentine Occupational Therapists certified in Sensory Integration. These professionals classified all 62 items of the QMSR with three labels that included "maintain" "modify" and "eliminate" and provided written suggestions for changes in their structure and composition. Additionally, they determined their inclusion in four categories: "Hypo-responsivity", "Hyper-responsivity", "Motor Planning" and "Social" and six sensory responsivity scales: "Visual", "Auditory", "Vestibular", "Taste/Smell", "Proprioceptive" and "Tactile". The final version of the QMRS and professional feedback on all its items, categories and scales is presented, together with suggestions for future studies that could potentially clarify its clinical utility and research applications.
\end{abstract}

\section{Key words:}

Autism, sensory responsivity, questionnaires, measurement instruments.

1 Ph.D., OTR/L Assistant Professor and Director of Technology. Nova Southeastern University (NSU). Health Professions Division. College of Health Care Sciences. Department of Occupational Therapy-Tampa. 3232 Queen Palm Drive. Tampa, Florida 33619-1311 * Tel: (813) 5745344 * Fax: (813) 574-5330. Email: greinoso@nova.edu 


\section{INTRODUCCIÓN}

La primera referencia a los problemas sensoriales en autismo fue publicada por Leo Kanner quién en 1943 los describió como causantes de comportamientos extraños así como también estrés causado por sensibilidades a ciertos aspectos del ambiente en varios de sus casos (Kanner, 1943). Las características sensoriales en los niños con autismo se han descripto de varios modos incluyendo hipo-respuesta, hiper-respuesta; intereses sensoriales, repeticiones y comportamientos de búsqueda y percepciones mejoradas (Ausderau et al., 2014; Ayres, 2005; Baranek, 2002; Baum, Stevenson, \& Wallace, 2015; E.I. Blanche \& Reinoso, 2007; CaseSmith, Weaver, \& Fristad, 2014; Case-Smith, Weaver, \& Fristad, 2015). Hipo-responsividad se refiere a una respuesta de intensidad disminuída o la falta de respuesta a los estímulos sensoriales. La hiper-responsividad se refiere a una respuesta de mayor amplitud o exagerada a los estímulos sensoriales. Los comportamientos de búsqueda han sido descriptos como situaciones en las cuales el individuo responde comprometiéndose con un aumento en la frecuencia, intensidad y duración en sus interacciones con información sensorial en diferentes acciones. Las percepciones mejoradas parecen relacionarse a mayor agudeza como por ejemplo en el reconocimiento de patrones visuales (Baranek, 2002; Kirby, Dickie, \& Baranek, 2015; Little, Ausderau, Sideris, \& Baranek, 2015; S.D. Tomchek \& Dunn, 2007). Estas características de procesamiento de la información sensorial son a menudo descriptas conjuntamente con problemas comportamentales. De este modo, algunas de estas diferencias dificultan el desempeño de acciones, tareas, actividades y ocupaciones como la higiene, el vestido y la alimentación así como también el desarroIlo de hábitos y rutinas que sostienen la participación y el bienestar en diferentes contextos como el hogar, la escuela y la comunidad.

Desde la primera mención de los problemas sensoriales en individuos con diagóstico de autismo se han publicado un gran número de estudios en la temática (Ashburner, Bennett, Rodger, \& Ziviani, 2014; Baker, Lane, Angley, \& Young, 2008; Baranek, 2002; Baranek et al., 2013; E.I. Blanche \& Reinoso, 2007; E. I. Blanche, Reinoso, Chang, \& Bodison, 2012; Case-Smith et al., 2014; Dunn, Saiter, \& Rinner, 2002; Greffou et al., 2012; James, Miller, Schaaf, Nielsen, \& Schoen, 2011; Lane, Dennis, \& Geraghty, 2011; Little et al., 2011;
Ludlow et al., 2014; O'Donnell, Deitz, Kartin, Nalty, \& Dawson, 2012; Paterson \& Peck, 2011; Stein et al., 2014; Talay-Ongan \& Wood, 2000; Wigham, Rodgers, South, McConachie, \& Freeston, 2015). Estos estudios han establecido los problemas sensoriales como un área de primordial importancia en el diagnóstico de individuos con autismo y su impacto en la vida personal, ocupacional, familiar y comunitaria.

Otro grupo de estudios se han abocado a la documentación de los diferentes patrones de respuesta a la información sensorial en individuos con diagnóstico de autismo en comparación con otras categorías diagnósticas y niños típicos (Baranek et al., 2013; E. I. Blanche, Reinoso, et al., 2012; Cheung \& Siu, 2009; Dunn et al., 2002; Kern et al., 2007; S. D. Tomchek, Huebner, \& Dunn, 2014). Las características sensoriales en los individuos con diagnóstico de autismo se miden con paradigmas de investigación en laboratorios por medio de diferentes métodos incluyendo respuestas electrodérmicas, electroencefalografía y potenciales evocados somatosensoriales, entre otros. En la práctica clínica se utilizan tests estandarizados, observaciones clínicas estructuradas y no estructuradas, instrumentos de observación y cuestionarios para los padres o personas que interactúan u observan al niño frecuentemente (Baranek et al., 2013; E. I. Blanche, Bodison, Chang, \& Reinoso, 2012; E.I. Blanche \& Reinoso, 2007; E.I. Blanche \& Reinoso, 2008) De este modo, toda evaluación diagnóstica incluye mediciones de la responsividad sensorial en niños con autismo.

Una nueva definición de autismo por la Asociación Psiquiátrica Americana ha incluído en su más reciente manual diagnóstico, el DSM-5, (American Psychiatric Associacion, 2013) la presencia de hipo e hiper responsividad como una característica de este diagnóstico. Estas características se ubican dentro de los patrones de comportamiento, intereses o actividades restringidos y repetitivos (RRB's). La inclusión de la responsividad sensorial como aspecto primario en el diagnóstico de autismo ha causado un renovado interés en la investigación relacionada a la evaluación de los problemas sensoriales. Un problema persiste, sin embargo, en países de habla hispana, y se centra fundamentalmente en la falta de acceso a instrumentos de medición desarrollados en la región. Resulta de particular importancia que los ítems de un cuestionario o instrumento de medición sean consistentes con los reportados en estudios internacionales pero además consensuados por profesiona- 
les que se desempeñan en los contextos geográficos y socio-culturales donde serán utilizados. Los Terapeutas Ocupacionales en Argentina hasta la fecha no cuentan con un instrumento desarrollado en el país siguiendo parámetros de investigación recomendados incluyendo un consenso estructurado de que ítems utilizar cuando se evalúan niños con diagnóstico de autismo. La situación de otros países de habla hispana es similar.

La metodología Delphi es de utilidad en instancias donde se busca consenso profesional estructurado. Delphi es un sistema para solicitar la opinión a un grupo de personas con particular interés en una temática (Brooks, 1979; Hasson, Keeney, \& Mckenna, 2000; Pill, 1971). Es por eso que permite desarrollar un consenso sistemático y progresivo en la selección y redacción final de un instrumento de medición. El propósito de este estudio es ampliar el desarrollo de un instrumento de medición desarrollado por el autor, un Cuestionario para Padres para la Medición de la Responsividad Sensorial en Niños con Diagnóstico de Autismo (CMRS y previamente conocido como PIS), para el estudio de la responsividad sensorial en niños con diagnóstico de autismo estableciendo parámetros de validez de constructo y contenido. Cuatro preguntas de investigación, estructuradas en 4 fases, guiaron el desarrollo e implementación del siguiente estudio de acuerdo a las procedimientos recomendados para investigaciones que utilizan la metodología Delphi:

1. Estudio 1: Revisión sistemática de la literatura. ¿Qué ítems sensoriales reportados en la literatura internacional tienen potencial para describir los problemas de responsividad sensorial en niños con diagnóstico de Autismo?

2. Estudio 2, Delphi 1: ¿Cuál es el grado de consenso para a) mantener, b) modificar o c) eliminar un grupo de ítems que forman parte de la versión piloto del CMRS, un cuestionario para padres para medir la responsividad sensorial en niños con autismo?

3. Estudio 3, Dephi 2: ¿Cuál es el grado de consenso para a) mantener o b) eliminar los ítems revisados en el estudio Delphi 1?

4. Estudio 4, Delphi 3: ¿Cual es el grado de consenso para clasificar el grupo final de ítems en cuatro categorías : a) Hipo-responsividad, b) Hiperresponsividad, c) Social y d) Planificación Motriz y dentro de seis escalas "Auditiva (AU)", "Gusto/ Olfato $(\mathrm{G} / \mathrm{O})$ ", "Propiocepción (PROP)", "Táctil (TAC)", "Vestibular (VEST)" y "Visual (VIS)"
Los estudios mencionados con anterioridad culminaron con la versión final del CMRS un cuestionario de 62 ítems, cuatro categorías y 6 escalas sensoriales que tiene como objetivo explorar la responsividad sensorial en niños con diagnóstico de autismo de habla hispana en la investigación y práctica clínica de Terapia Ocupacional y otras disciplinas que trabajan con individuos con este diagnóstico.

\section{Metodo}

\section{Participantes}

El presente estudio utilizó como criterio de inclusión a todos los terapeutas ocupacionales en Argentina que hubiesen completado los niveles de formación 3 y 4 en la certificación Argentina de Integración Sensorial (Reinoso, 2007/2015). Una reunión realizada en la ciudad de Buenos Aires en Mayo del 2015 en modalidad presencial y virtual proporcionó información adicional a los participantes en relación a la metodología del estudio y obtuvo su consentimiento informado. Se diseñaron cuatro estudios siguiendo la metodología de investigación de entrevistas y estudios Delphi sugerida por difererentes autores (Andrews, Nonnecke, \& Preece, 2003; Couper, 2000; Fowler \& Floyd, 1995; Greatorex, 2000; Hsu \& Sandord, 2007; Okoli \& Pawlowski, 2004; Weible \& Wallace, 1999). Todas las entrevistas fueron vinculadas a una base de datos y administradas de manera anónima y enviadas a los participantes seleccionados. La muestra de los estudios incluyeron a ochenta y siete $(\mathrm{N}=87)$, setenta y tres $(\mathrm{N}=73)$ y sesenta $(\mathrm{N}=60)$ Terapeutas Ocupacionales que participaron en los estudios Delphi 2, 3 y 4 respectivamente.

\section{Revisión de la Literatura}

Una revisión sistemática de la literatura se completó para la elaboración de los ítems pilotos del CMRS y refinamiento de su precursor un cuestionario denominado PIS (procesamiento de información sensorial no publicado) cuyo proceso se describe en detalle en la sección de resultados. 


\section{Estudios Delphi}

Se completaron 3 estudios Delphi. El estudio 2 o Delphi $1(\mathrm{~N}=87)$ se centró en solicitar a los participantes "Mantener", "Eliminar" o "Modificar" los 62 ítems presentados e identificados en la revisión de la literatura. Un espacio de texto se presentó en la opción "Modificar" para que cada participante pudiera expresar como ese ítem debería redactarse para su mejor comprensión o utilización clínica. El estudio 3 o Delphi $2(\mathrm{~N}=73)$ se centró en revisar los ítems que incluyeron todas las modificaciones realizadas en el estudio anterior. Las opciones de este estudio sólo incluyeron las opciones de "mantener" o "eliminar" para cada ítem. El estudio 4 o Delphi $3(\mathrm{~N}=60)$ invitó a los participantes a clasificar cada ítem. Dos sistemas de clasificación fueron utilizados. Primero se solicitó clasificar el ítem como perteneciente a la categoría de "Hipo-responsividad (HIPO)", "Hiper-responsivity (HIPER)", "Social (SOC)" y "Planificación Motriz (PM)". El mismo ítem se repitió y se solicitó que el profesional lo clasificara como perteneciente a una de seis escalas: "Auditiva $(A U)$ ", "Gusto/Olfato (G/O)", "Propiocepción (PROP)", "Táctil (TAC)", "Vestibular (VEST)" y "Visual (VIS)". Ambas clasificaciones fueron de opciones forzadas, es decir, no se permitió clasificar un ítem como perteneciente a más de una categoría o escala.

\section{Resultados}

\section{Estudio 1: Revisión Sistemática de la Literatura}

La revisión de la literatura fue completada por el autor y se llevó a cabo utilizando las siguientes bases de datos: Taylor \& Francis Online, CINAHL Complete, SAGE Journals, SpringerLink \& Journal of Autism and Developmental Disorders, ScienceDirect, Medline, ProQuest and EbscoHost. Palabras clave y términos MESH incluyeron combinaciones las siguientes combinaciones: "autism", "sensory", "sensory processing", "taste", "smell", "olfactory", "vision", "auditory", "sound", "tactile defensiveness", "oral", "food", "sticky substances", "peripheral vision", "copying forms", "vestibular", "proprioceptive", "mealtime", "food choices" y "transportation". Filtros para "humanos" y períodos entre los años 2000 y 2015 fueron seleccionados. Los artículos identificados fueron analizados y revisados para determinar su relación al tema de investigación. Las búsquedas y permutaciones resultaron en 3.675 artículos. Todos los títulos de estos artículos fueron revisados y clasificados en relación a su relevancia al tema de investigación. De los títulos revisados se seleccionaron 54 artículos que fueron revisados en su totalidad. De acuerdo a las descripciones de la literatura y la experiencia del investigador se diseñaron 177 ítems. Subsecuentes revisiones culminaron en el conjunto de los 62 ítems finales que se utilizaron en los estudios Deplhi 1, 2 y 3. La Tabla 1 enumera los ítems finales del CMRS y la literatura que apoyó su incorporación. 
Tabla 1

Las seis escalas del CMRS, su descripción y autores que apoyan su contenido en diferentes estudios.

\begin{tabular}{|c|c|c|c|}
\hline Escala & Item \# & Descripción & Autores \\
\hline \multirow{11}{*}{ VIS } & $\# 1^{*}$ & $\begin{array}{l}\text { Parece ser sensible a la luz (ej. cierra } \\
\text { los ojos o baja la mirada al salir al sol } \\
\text { o en lugares con luz artificial) }\end{array}$ & $\begin{array}{c}\text { (Ashburner, Bennett, Rodger, \& Ziviani, 2013; Litt- } \\
\text { le et al., 2011; Marco, Hinkley, Hill, \& Nagarajan, } \\
\text { 2011; Tomchek \& Dunn, 2007; Tomchek, } \\
\text { Huebner, \& Dunn, 2014) }\end{array}$ \\
\hline & $\# 2$ & $\begin{array}{l}\text { Se acerca demasiado al papel o super- } \\
\text { ficie cuando dibuja, copia o pinta }\end{array}$ & (Lipsky, 2011; Thorpe \& Crispin, n.d.) \\
\hline & $\# 3$ & $\begin{array}{l}\text { Mueve sus manos/dedos cerca de sus } \\
\text { ojos }\end{array}$ & $\begin{array}{l}\text { (Marco et al., 2011; Ozonoff, Williams, } \\
\text { \& Landa, 2005) }\end{array}$ \\
\hline & $\# 4$ & $\begin{array}{c}\text { Coloca sus ojos hacia los lados (mira } \\
\text { de costado o de reojo) }\end{array}$ & (Lipsky, 2011; Thorpe \& Crispin, n.d.) \\
\hline & $\# 5$ & $\begin{array}{l}\text { Tiene dificultad con actividades visu- } \\
\text { ales para su edad (ej. rompecabezas, } \\
\text { construir con bloques, copiar formas) }\end{array}$ & $\begin{array}{c}\text { (Baker, Lane, Angley, \& Young, 2008; Mayes \& } \\
\text { Calhoun, 2003) }\end{array}$ \\
\hline & $\# 6$ & $\begin{array}{l}\text { Parece no notar algunos objetos en el } \\
\text { ambiente (ej. Se tropieza con objetos) }\end{array}$ & (Davis et al., 2006; Thorpe \& Crispin, n.d.) \\
\hline & $\# 7$ & $\begin{array}{l}\text { Presta excesiva atención a ciertas imá- } \\
\text { genes, letras, números o símbolos }\end{array}$ & $\begin{array}{l}\text { (Kirby, Little, Schultz, Baranek, 2015; Talay-Ongan } \\
\text { \& Wood, 2000; Zwaigenbaum, 2005) }\end{array}$ \\
\hline & $\# 8$ & $\begin{array}{c}\text { Pestañea o se frota los ojos con } \\
\text { frecuencia }\end{array}$ & $\begin{array}{l}\text { (Kirby, Dickie, \& Baranek, 2014; } \\
\text { Oristaglio, et al., 2013) }\end{array}$ \\
\hline & $\# 9$ & $\begin{array}{l}\text { Se distrae con información visual (ej. } \\
\text { necesita estar en un ambiente libre de } \\
\text { distracciones para poner atención o } \\
\text { aprender algo nuevo) }\end{array}$ & (Al Abdulmohsen \& Kruger, 2011; Lipsky, 2011) \\
\hline & $\# 10$ & $\begin{array}{l}\text { Inclina su cabeza cuando mira a } \\
\text { algunos objetos, imágenes o personas }\end{array}$ & $\begin{array}{c}\text { (Bagatell, 2012; Brereton, Tonge, Mackinnon, \& } \\
\text { Einfeld, 2002) }\end{array}$ \\
\hline & $\# 11$ & $\begin{array}{l}\text { Prefiere comidas de colores } \\
\text { específicos }\end{array}$ & $\begin{array}{c}\text { (Lockner, Crowe, \& Skipper, 2008; } \\
\text { Provost et al., 2010) }\end{array}$ \\
\hline
\end{tabular}




\begin{tabular}{|c|c|c|c|}
\hline Escala & Item \# & Descripción & Autores \\
\hline \multirow{9}{*}{$\mathrm{G} / \mathrm{O}$} & $\# 12$ & $\begin{array}{l}\text { Muestra reacciones exageradas a algu- } \\
\text { nos sabores o aromas }\end{array}$ & $\begin{array}{c}\text { (DeMand, Johnson, \& Foldes, 2015; Thorpe \& } \\
\text { Crispin, n.d.) }\end{array}$ \\
\hline & $\# 13$ & $\begin{array}{l}\text { Algunas comidas, olores o sabores le } \\
\text { producen arcadas y/o ganas de vomitar }\end{array}$ & $\begin{array}{c}\text { (DeMand et al., 2015; Noor Safiza, \& Le Couteur, } \\
\text { 2015) }\end{array}$ \\
\hline & $\# 14$ & $\begin{array}{l}\text { Tiene preferencias muy marcadas por } \\
\text { sabores particulares }\end{array}$ & $\begin{array}{l}\text { (DeMand et al., 2015; Miller, Anzalone, Lane, } \\
\text { Cermak, \& Osten, 2007; Paterson, \& Peck, 2011; } \\
\text { Tomchek, Huebner \& Dunn, 2014) }\end{array}$ \\
\hline & $\# 15$ & $\begin{array}{c}\text { Tiende a oler objetos, materiales y } \\
\text { texturas mucho más que otros niños } \\
\text { de su edad }\end{array}$ & $\begin{array}{l}\text { (Baker et al., 2008; Brereton et al., 2002; Kirby et } \\
\text { al., 2015; Little et al., 2011) }\end{array}$ \\
\hline & $\# 16$ & $\begin{array}{l}\text { Come o mastica objetos no comesti- } \\
\text { bles (ej. tierra, pintura, plastilina, lápiz, } \\
\text { cuello de la ropa) }\end{array}$ & $\begin{array}{c}\text { (Brereton et al., 2002; Kerwin, Eicher, \& } \\
\text { Gelsinger, 2005;Kirby et al., 2015; Little et al., } \\
\text { 2011; Noor Safiza, \& Le Couteur, 2015; } \\
\text { Talay-Ongan \& Wood, 2000) }\end{array}$ \\
\hline & $\# 17$ & $\begin{array}{l}\text { Le desagrada el olor de los perfumes, } \\
\text { desodorantes o artículos de limpieza }\end{array}$ & $\begin{array}{c}\text { (Ashburner et al., 2013; Legiša, Messinger, Kermol, } \\
\text { \& Marlier, 2013) }\end{array}$ \\
\hline & $\# 18$ & $\begin{array}{l}\text { El aroma o gusto de las comidas limita } \\
\text { su dieta significativamente }\end{array}$ & $\begin{array}{c}\text { (Ashburner et al., 2013; Paterson, \& Peck, 2011; } \\
\text { Tomchek \& Dunn, 2007; Tomchek, Huebner \& } \\
\text { Dunn, 2014) }\end{array}$ \\
\hline & $\# 19$ & Tiene una dieta limitada & $\begin{array}{l}\text { (Ashburner et al., 2013; Brereton et al., 2002; } \\
\text { Crasta et al., 2014; DeMand et al., 2015; Kern et } \\
\text { al., 2001; Noor Safiza, \& Le Couteur, 2015; } \\
\text { Paterson, \& Peck, 2011; Talay-Ongan \& Wood, } \\
\text { 2000; Tomchek \& Dunn, 2007; Tomchek, Hueb- } \\
\text { ner \& Dunn, 2014) }\end{array}$ \\
\hline & $\# 20$ & $\begin{array}{l}\text { Se da cuenta de la presencia de olores } \\
\text { fuertes en el ambiente }\end{array}$ & $\begin{array}{l}\text { (Dudova et al., 2011; Talay-Ongan } \\
\text { \& Wood, 2000) }\end{array}$ \\
\hline
\end{tabular}




\begin{tabular}{|c|c|c|c|}
\hline Escala & Item \# & Descripción & Autores \\
\hline \multirow{10}{*}{$\mathrm{AU}$} & $\# 21$ & $\begin{array}{l}\text { Llora o se molesta con los sonidos de } \\
\text { artículos del hogar (ej. aspiradora, seca- } \\
\text { dor de pelo, radio, timbre, batidora) }\end{array}$ & $\begin{array}{l}\text { (Ashburner et al., 2013; Ludlow et al., 2014; Stie- } \\
\text { gler \& Davis, 2010; Talay-Ongan \& Wood, 2000) }\end{array}$ \\
\hline & $\# 22$ & $\begin{array}{l}\text { El volumen de su voz es muy alto o } \\
\text { muy bajo }\end{array}$ & $\begin{array}{c}\text { (Alcántara, Weisblatt, Moore, \& Bolton, 2004; } \\
\text { Ashburner et al., 2013) }\end{array}$ \\
\hline & $\# 23$ & $\begin{array}{l}\text { Responde inmediatamente a su } \\
\text { nombre }\end{array}$ & $\begin{array}{l}\text { (Ashburner et al., 2013; Ashburner, Ziviani, \& } \\
\text { Rodger, 2008; Little et al., 2011; Tomchek, \& } \\
\text { Dunn, 2007; Tomchek, et al., 2014) }\end{array}$ \\
\hline & $\# 24$ & $\begin{array}{l}\text { Se confunde cuando se le pide algo } \\
\text { verbalmente (ej. tiene dificultades } \\
\text { siguiendo instrucciones verbales) }\end{array}$ & $\begin{array}{c}\text { (Alcántara, et al., 2004; Ashburner et al., 2008; } \\
\text { Ludlow, et al., 2014) }\end{array}$ \\
\hline & $\# 25$ & $\begin{array}{l}\text { Se cubre los oídos en presencia de } \\
\text { sonidos que no molestan a otras per- } \\
\text { sonas (ej. campana, sirena, silbato, } \\
\text { timbre, artículos electrodomésticos) }\end{array}$ & $\begin{array}{c}\text { (Ashburner et al., 2013; Brereton et al., 2002; } \\
\text { Lucker, 2013; Mulligan \& White, 2012; Stiegler \& } \\
\text { Davis, 2010; Tomchek \& Dunn, 2007; Tomchek et } \\
\text { al., 2014) }\end{array}$ \\
\hline & $\# 26$ & Repite frases, palabras, o sonidos & $\begin{array}{c}\text { (Dunn, Saiter, \& Rinner, 2002; Wigham, Rodgers, } \\
\text { McConachie, \& Freeston, 2015) }\end{array}$ \\
\hline & $\# 27$ & $\begin{array}{l}\text { Intenta controlar el volumen de dife- } \\
\text { rentes sonidos (radio, televisión, voz, } \\
\text { música) }\end{array}$ & $\begin{array}{c}\text { (Ashburner, et al., 2008; Stiegler \& Davis, 2010; } \\
\text { Talay-Ongan \& Wood, 2000) }\end{array}$ \\
\hline & $\# 28$ & $\begin{array}{l}\text { Evita y se desorganiza en ambientes } \\
\text { con muchos estímulos (shopping, su- } \\
\text { permercado, tienda, transporte públi- } \\
\text { co, fiestas de cumpleaños, restaurant) }\end{array}$ & $\begin{array}{l}\text { (Ashburner et al., 2008; Ashburner et al., 2013; } \\
\text { Baker et al., 2008; Kern et al., 2001; Ludlow et al., } \\
\text { 2014; Stiegler \& Davis, 2010; Tomchek, Huebner } \\
\text { \& Dunn, 2014) }\end{array}$ \\
\hline & \multicolumn{3}{|c|}{$\# 29$} \\
\hline & $\# 30$ & $\begin{array}{c}\text { Hace mucho ruido con objetos, } \\
\text { juguetes o materiales (e.j. los golpea, } \\
\text { murmura) }\end{array}$ & (Ashburner et al., 2008; Thorpe \& Crispin, n.d.) \\
\hline
\end{tabular}




\begin{tabular}{|c|c|c|c|}
\hline Escala & Item \# & Descripción & Autores \\
\hline \multirow{10}{*}{ VEST } & $\# 31$ & $\begin{array}{l}\text { Llora o parece sentir naúseas luego de } \\
\text { viajes en el auto o transporte público }\end{array}$ & $\begin{array}{l}\text { (Thorpe \& Crispin, n.d.; Yonkman, Lawler, Talty, } \\
\text { O'Neil \& Bull, 2013) }\end{array}$ \\
\hline & $\# 32$ & $\begin{array}{l}\text { Se resiste a utilizar equipos con mov- } \\
\text { imiento (ej. ascensor, escalera mecáni- } \\
\text { ca, calesita, parque de diversiones, } \\
\text { sube y baja) }\end{array}$ & $\begin{array}{c}\text { (Lane, Dennis, \& Geraghty, 2011; Thorpe \& } \\
\text { Crispin, n.d.) }\end{array}$ \\
\hline & $\# 33$ & $\begin{array}{l}\text { Le agradan las actividades que in- } \\
\text { cluyen movimientos intensos (e.j. ser } \\
\text { lanzado en el aire, parques de diver- } \\
\text { siones, trampolín, correr) }\end{array}$ & (Ashburner, Bennet, Rodger \& Ziviani, 2013) \\
\hline & $\# 34$ & $\begin{array}{l}\text { Busca apoyarse cuando está en la } \\
\text { mesa (e.j. sostiene la cabeza con sus } \\
\text { manos, se apoya en otras personas) }\end{array}$ & $\begin{array}{l}\text { (Lane, Dennis \& Geragthy, 2011; Tomchek, Hueb- } \\
\text { ner \& Dunn, 2014) }\end{array}$ \\
\hline & $\# 35$ & $\begin{array}{l}\text { Le desagradan los cambios de posición } \\
\text { (ej. moverse en la silla, saltar, subir o } \\
\text { bajar escaleras, treparse, hamacarse) }\end{array}$ & (Thorpe \& Crispin, n.d.) \\
\hline & $\# 36$ & $\begin{array}{l}\text { Parece ansioso/a en el parque y du- } \\
\text { rante actividades que requieren saltar, } \\
\text { girarse, treparse, bajarse o subirse }\end{array}$ & (Lane, Dennis \& Geragthy, 2011; Poon, 2012) \\
\hline & $\# 37$ & Se cae con frecuencia & $\begin{array}{c}\text { (Lane, Dennis \& Geragthy, 2011; Thorpe \& } \\
\text { Crispin, n.d.) }\end{array}$ \\
\hline & $\# 38$ & $\begin{array}{l}\text { Tiene mucha más energía que otros } \\
\text { niños de su edad cuando se despierta } \\
\text { (ej. salta en la cama, corre, se mueve } \\
\text { excesivamente, es muy activo) }\end{array}$ & $\begin{array}{l}\text { (Dunn, Saiter, Rinner, 2002; O’Donnell, Deitz, Kar- } \\
\text { tin, Nalty \& Dawson, 2012; Thorpe \& Crispin, n.d.) }\end{array}$ \\
\hline & $\# 39$ & $\begin{array}{l}\text { Tiene dificultad con actividades que } \\
\text { requieren equilibrio (triciclo, bicicleta, } \\
\text { juegos de equilibrio o balancearse) }\end{array}$ & (Kern et al., 2007) \\
\hline & $\# 40$ & $\begin{array}{l}\text { Le es difícil atrapar pelotas cuando se } \\
\text { compara con niños de su edad }\end{array}$ & $\begin{array}{c}\text { (Weimer, Schatz, Lincoln, Ballantyne, \& Trauner, } \\
\text { 2001) }\end{array}$ \\
\hline
\end{tabular}




\begin{tabular}{|c|c|c|c|}
\hline Escala & Item \# & Descripción & Autores \\
\hline \multirow{12}{*}{ TAC } & $\# 41$ & $\begin{array}{l}\text { Le molesta jugar con pintura de dedos, } \\
\text { crema de afeitar u otros materiales } \\
\text { blando, pegajosos o granulosos }\end{array}$ & $\begin{array}{l}\text { (Kumar, lbagere, Singh, Odo, \& Aro, 2015; } \\
\text { Talay-Ongan \& Wood, 2000) }\end{array}$ \\
\hline & $\# 42$ & $\begin{array}{l}\text { Evita alimentos blandos como } \\
\text { pure, yogurt o flan }\end{array}$ & $\begin{array}{l}\text { (Smith, Roux, Naidoo, \& Venter, 2005; } \\
\text { Talay-Ongan \& Wood, 2000) }\end{array}$ \\
\hline & $\# 43$ & \begin{tabular}{|c}
$\begin{array}{c}\text { Evita alimentos que requieren masticar } \\
\text { (ej. carne) }\end{array}$ \\
\end{tabular} & (DeMand et al., 2015; Smith et al., 2005) \\
\hline & $\# 44$ & $\begin{array}{c}\text { Frota sus manos, cara, cuerpo en su- } \\
\text { perficies, texturas u objetos }\end{array}$ & $\begin{array}{l}\text { (Kirby, Little, Schultz \& Baranek, 2015; Thorpe } \\
\text { \& Crispin, n.d.) }\end{array}$ \\
\hline & $\# 45$ & $\begin{array}{c}\text { Evita el contacto físico con algunas } \\
\text { superficies, objetos o texturas }\end{array}$ & $\begin{array}{c}\text { (Dunn, Saiter \& Rinner, 2002; Kumar, Ibagere, } \\
\text { Singh \& Odo, 2015) }\end{array}$ \\
\hline & $\# 46$ & $\begin{array}{l}\text { Toca objetos y superficies con su } \\
\text { lengua, labios, boca, o cara }\end{array}$ & $\begin{array}{c}\text { (Baker, Lane, Angley \& Young, 2008; Brereton, } \\
\text { Tonge, Mackinnon \& Einfeld, 2002; Thorpe \& } \\
\text { Crispin, n.d.) }\end{array}$ \\
\hline & $\# 47$ & $\begin{array}{l}\text { Presenta estrés durante actividades de } \\
\text { higiene o arreglo personal (ej. cepil- } \\
\text { lado de dientes, lavado de cara, cor- } \\
\text { tado de uñas, lavado de cabello, baño, } \\
\text { cortado de cabello) }\end{array}$ & $\begin{array}{l}\text { (Ashburner et al., 2013; Kern et al., 2001; Little et } \\
\text { al., 2011; Talay-Ongan \& Wood, 2000; Tomchek } \\
\text { et al., 2014) }\end{array}$ \\
\hline & $\# 48$ & Lleva objetos no comestibles a la boca & $\begin{array}{c}\text { (Dunn, Saiter \& Rinner 2002; Thorpe \& Crispin, } \\
\text { n.d.) }\end{array}$ \\
\hline & $\# 49$ & $\begin{array}{l}\text { Se niega a utilizar algunas prendas de } \\
\text { vestir (ej. Sweaters de lana, nylon, etc.) }\end{array}$ & $\begin{array}{l}\text { (Ashburner, Better, Rodger \& Ziviani, 2013; Bre- } \\
\text { reton, Tonge, Mackinnon \& Einfeld, 2002; Dunn, } \\
\text { Saiter \& Rinner, 2002; Kern et al., 2001; Talay- } \\
\text { Ongan \& Wood, 2000) }\end{array}$ \\
\hline & $\# 50$ & $\begin{array}{c}\text { Tiene dificultad con el espacio per- } \\
\text { sonal (ej. toca o se acerca demasiado a } \\
\text { otros niños o personas) }\end{array}$ & $\begin{array}{c}\text { (Ashburner, Bennet, Rodger \& Ziviani, 2013; Lip- } \\
\text { sky, 2011; Talay-Ongan \& Wood, 2000) }\end{array}$ \\
\hline & $\# 51$ & $\begin{array}{l}\text { Parece no sentir el dolor de la misma } \\
\text { forma que otros niños de su edad }\end{array}$ & $\begin{array}{l}\text { (Brereton, Tonge, Mackinnon \& Einfel, 2002; Kirby } \\
\text { et al., 2015; Lane, Young, Baker, \& Angley, 2010; } \\
\text { Little et al., 2011; Talay-Ongan \& Wood, 2000) }\end{array}$ \\
\hline & $\# 52$ & $\begin{array}{c}\text { Le desagrada el contacto o proximi- } \\
\text { dad con otras personas (e.j. durante el } \\
\text { juego en el salón de clase, formando } \\
\text { una fila, en el parque) }\end{array}$ & $\begin{array}{l}\text { (Brereton et al., 2002; Dunn, Saiter \& Rinner, 2002; } \\
\text { Kern et al., 2001; Talay-Ongan \& Wood, 2000) }\end{array}$ \\
\hline
\end{tabular}




\begin{tabular}{|c|c|c|c|}
\hline Escala & Item \# & Descripción & Autores \\
\hline \multirow{10}{*}{ PROP } & $\# 53$ & $\begin{array}{l}\text { Rompe juguetes, objetos y materiales } \\
\text { con facilidad }\end{array}$ & $\begin{array}{l}\text { (Blanche, Reinoso, Chang \& Bodison, 2012; Brere- } \\
\text { ton, Tonge, Mackinnon \& Einfel, 2002; Poon, 2012) }\end{array}$ \\
\hline & $\# 54$ & $\begin{array}{l}\text { Tiene dificultades para medir su fuerza } \\
\text { o utiliza demasiada o muy poca fuerza } \\
\text { con algunos objetos (e.j. lápiz, crayón, } \\
\text { tenedor, cuchara, cerrar la puerta) }\end{array}$ & $\begin{array}{l}\text { (Blanche, Reinoso, Chang \& Bodison, 2012; } \\
\text { Brereton et al., 2002; Thorpe \& Crispin, n.d.) }\end{array}$ \\
\hline & $\# 55$ & $\begin{array}{l}\text { Busca actividades como moverse, cor- } \\
\text { rer, saltar, treparse, lanzarse, colgarse, } \\
\text { patear más que otros niños de su edad }\end{array}$ & $\begin{array}{c}\text { (Ashburner, Bennet, Rodger \& Ziviani, 2013; } \\
\text { Blanche, Reinoso, Chang \& Bodison, 2012; } \\
\text { Dawson \& Watling, 2000; Tonge, Mackinnon \& } \\
\text { Einfeld, 2002; Talay-Ongan \& Wood, 2000; Tom- } \\
\text { chek, Huebner \& Dunn 2014) }\end{array}$ \\
\hline & $\# 56$ & $\begin{array}{l}\text { Sus músculos parecen blandos } \\
\text { y débiles }\end{array}$ & $\begin{array}{c}\text { (Baranek, 2002; Tomchek, Huebner } \\
\text { \& Dunn 2014) }\end{array}$ \\
\hline & $\# 57$ & $\begin{array}{l}\text { Tiende a utilizar movimientos muy am- } \\
\text { plios y bruscos cuando no se requieren }\end{array}$ & $\begin{array}{l}\text { (Brereton, Tonge, Mackinnon, Einfeld, 2002; Lip- } \\
\text { sky, 2011) }\end{array}$ \\
\hline & $\# 58$ & $\begin{array}{c}\text { Mastica objetos (e.j. cuello de su cami- } \\
\text { sa, lápiz, crayón, puño de su manga, } \\
\text { goma de borrar) }\end{array}$ & $\begin{array}{l}\text { (Brereton, Tonge, Mackinnon, Einfeld, 2002; Thor- } \\
\text { pe \& Crispin, n.d.) }\end{array}$ \\
\hline & $\# 59$ & $\begin{array}{c}\text { Es brusco en sus interacciones con } \\
\text { otros niños (ej. golpea, empuja, aprie- } \\
\text { ta, pellizca, muerde) }\end{array}$ & $\begin{array}{c}\text { (Brereton, Tonge, Mackinnon, Einfeld, 2002; } \\
\text { Poon, 2012) }\end{array}$ \\
\hline & $\# 60$ & $\begin{array}{l}\text { Es torpe en comparación a niños de } \\
\text { su eda }\end{array}$ & $\begin{array}{l}\text { (Al Abdulmohsen \& Kruger, 2011; Dawson \& } \\
\text { Watling, 2000; Dunn, Saiter \& Rinner, 2002; } \\
\text { Weimer, Schatz, Lincoln, Ballantyne \& Trauner, } \\
\text { 2001) }\end{array}$ \\
\hline & $\# 61$ & Camina en puntas de pie & $\begin{array}{l}\text { (Baranek, 2002; Blanche, Reinoso, Chang \& Bodi- } \\
\text { son, 2012; Brereton, Tonge, Mackinnon \& Einfeld, } \\
\text { 2002; Dzuik, Larson, Apostu, Mahone, Denckla \& } \\
\text { Mostofsky, 2007; Piek \& Dyck, 2004) }\end{array}$ \\
\hline & $\# 62$ & $\begin{array}{c}\text { Se tropieza y choca con objetos en el } \\
\text { ambiente (ej. personas, muebles, } \\
\text { marcos de la puerta) }\end{array}$ & $\begin{array}{c}\text { (Ayres, 2011; Blanche, Reinoso, Chang \& Bodi- } \\
\text { son, 2012; Green, Baird, Barnett, Henderson, } \\
\text { Huber \& Henderson, 2002; Jansiewicz, Goldbert, } \\
\text { Newschaffer, Denckla, Landa \& Mostofsky, 2006; } \\
\text { Minshew, Sung, \& Jones, 2004; Molloy, Dietrich } \\
\text { \& Bhattacharya, 2003; Sacrey, Germani, Bryson, } \\
\text { \& Zwaigenbaum, 2014) }\end{array}$ \\
\hline
\end{tabular}

Nota. VIS = Escala Visual; G/O = Escala Gusto/Olfato; AU= Escala Auditiva; VES = Escala Vestibular; TAC = Escala Táctil; PROP = Escala Proprioceptiva; $\left(^{*}\right)=$ número de ítems. 


\section{Estudio 2, Delphi 1}

Los ochenta y siete terapeutas ocupacionales $(\mathrm{N}=87)$ que participaron en este estudio revisaron 62 ítems. El rango en el nivel de acuerdo para estos ítems en la categoría "mantener" fue entre $51.72 \%$ y $100 \%$. El nivel de acuerdo para los ítems en la categoría "eliminar" fue entre $0 \%$ y $18.39 \%$. El nivel de acuerdo para los ítems en la categoría "modificar" fue entre $0 \%$ y $34.48 \%$. Un nivel de $80 \%$ y $1 \%$ fueron establecidos a priori para eliminar y revisar un ítem, respectivamente. La tabla 2 ilustra el ranking de los 87 participantes en cada ítem del CMRS y su clasificación para revisarse o modificarse. De este modo, 59 ítems fueron revisados con correcciones menores (e.j. tiempo verbal, sinónimo) y 14 fueron modificados en su redacción para mejor comprensión.

Tabla 2

Ranking (N=87) de los ítems del CMRS; Estudio 2 Delphi 1

\begin{tabular}{|c|c|c|c|c|c|c|}
\hline Estudio & Item & Mantener & Eliminar & Modificar & Revisado & Modificado \\
\hline \multirow[t]{26}{*}{2} & $\# 1$ & $80(91.95 \%)$ & $0(0 \%)$ & $7(8.05 \%)$ & $*$ & \\
\hline & $\# 2$ & $53(60.92 \%)$ & $4(4.60 \%)$ & $30(34.48 \%)$ & $*$ & + \\
\hline & $\# 3$ & $77(88.51 \%)$ & $3(3.45 \%)$ & $7(8.05 \%)$ & $*$ & \\
\hline & $\# 4$ & $45(51.72 \%)$ & $12(13.79)$ & $30(34.48 \%)$ & $*$ & $t$ \\
\hline & $\# 5$ & $73(83.91 \%)$ & $4(4.60 \%)$ & $10(11.49 \%)$ & $*$ & \\
\hline & $\# 6$ & $77(88.51 \%)$ & $1(1.15 \%)$ & $9(10.34 \%)$ & $*$ & $t$ \\
\hline & $\# 7$ & $82(94.25 \%)$ & $2(2.30 \%)$ & $3(3.45 \%)$ & $*$ & \\
\hline & $\# 8$ & $78(89.66 \%)$ & $7(8.05 \%)$ & $2(2.30 \%)$ & $*$ & \\
\hline & $\# 9$ & $77(88.51 \%)$ & $2(2.30 \%)$ & $8(9.20 \%)$ & $*$ & \\
\hline & $\# 10$ & $78(89.66 \%)$ & $4(4.60 \%)$ & $5(5.75 \%)$ & $*$ & \\
\hline & $\# 11$ & $72(82.76 \%)$ & $5(5.75 \%)$ & $10(11.49 \%)$ & $*$ & $t$ \\
\hline & $\# 12$ & $78(89.66 \%)$ & $5(5.75 \%)$ & $4(4.60 \%)$ & $*$ & \\
\hline & $\# 13$ & $83(95.40 \%)$ & $2(2.30 \%)$ & $2(2.30 \%)$ & $*$ & \\
\hline & $\# 14$ & $81(93.10 \%)$ & $2(2.30 \%)$ & $4(4.60 \%)$ & $*$ & \\
\hline & $\# 15$ & $79(90.80 \%)$ & $1(1.15 \%)$ & $7(8.05 \%)$ & * & \\
\hline & $\# 16$ & $87(100 \%)$ & $0(0 \%)$ & $0(0 \%)$ & & \\
\hline & $\# 17$ & $75(86.21 \%)$ & $8(9.20 \%)$ & $4(4.60 \%)$ & $*$ & \\
\hline & $\# 18$ & $74(85.06 \%)$ & $4(4.60 \%)$ & $9(10.34 \%)$ & $*$ & \\
\hline & $\# 19$ & $69(79.31 \%)$ & $7(8.05 \%)$ & $11(12.64 \%)$ & $*$ & $t$ \\
\hline & $\# 20$ & $62(71.26 \%)$ & $7(8.05 \%)$ & $18(20.69 \%)$ & $*$ & $t$ \\
\hline & $\# 21$ & $79(90.80 \%)$ & $1(1.15 \%)$ & $7(8.05 \%)$ & $*$ & \\
\hline & $\# 22$ & $82(94.25 \%)$ & $1(1.15 \%)$ & $4(4.60 \%)$ & $*$ & \\
\hline & $\# 23$ & $79(90.80 \%)$ & $4(4.60 \%)$ & $4(4.60 \%)$ & $*$ & \\
\hline & $\# 24$ & $78(89.66 \%)$ & $0(0 \%)$ & $9(10.34 \%)$ & $*$ & \\
\hline & $\# 25$ & $82(94.25 \%)$ & $3(3.45 \%)$ & $2(2.30 \%)$ & $*$ & \\
\hline & $\# 26$ & $81(93.10 \%)$ & $1(1.15 \%)$ & $5(5.75 \%)$ & $*$ & \\
\hline
\end{tabular}




\begin{tabular}{|c|c|c|c|c|c|c|}
\hline Estudio & Item & Mantener & Eliminar & Modificar & Revisado & Modificado \\
\hline & $\# 27$ & $80(91.95 \%)$ & $4(4.60 \%)$ & $3(3.45 \%)$ & $*$ & \\
\hline & $\# 28$ & $76(87.36 \%)$ & $0(0 \%)$ & $11(12.64 \%)$ & $*$ & \\
\hline & $\# 29$ & $80(91.95 \%)$ & $3(3.45 \%)$ & $4(4.60 \%)$ & $*$ & \\
\hline & $\# 30$ & $76(87.36 \%)$ & $3(3.45 \%)$ & $8(9.20 \%)$ & $*$ & + \\
\hline & $\# 31$ & $79(90.80 \%)$ & $1(1.15 \%)$ & $7(8.05 \%)$ & $*$ & \\
\hline & $\# 32$ & $80(91.95 \%)$ & $0(0 \%)$ & $7(8.05)$ & $*$ & \\
\hline & $\# 33$ & $84(96.55)$ & $0(0 \%)$ & $3(3.45 \%)$ & $*$ & \\
\hline & $\# 34$ & $79(90.80 \%)$ & $1(1.15 \%)$ & $7(8.05 \%)$ & $*$ & $t$ \\
\hline & $\# 35$ & $76(87.36 \%)$ & $4(4.60 \%)$ & $7(8.05 \%)$ & $*$ & \\
\hline & $\# 36$ & $76(87.36 \%)$ & $0(0 \%)$ & $11(12.64 \%)$ & $*$ & \\
\hline & $\# 37$ & $84(96.55 \%)$ & $2(2.30 \%)$ & $1(1.15 \%)$ & $*$ & \\
\hline & $\# 38$ & $81(93.10 \%)$ & $2(2.30 \%)$ & $4(4.60 \%)$ & $*$ & \\
\hline & $\# 39$ & $82(94.25 \%)$ & $1(1.15 \%)$ & $4(4.60 \%)$ & $*$ & \\
\hline & $\# 40$ & $82(94.25 \%)$ & $0(0 \%)$ & $5(5.75 \%)$ & $*$ & \\
\hline & $\# 41$ & $77(88.51 \%)$ & $0(0 \%)$ & $10(11.49 \%)$ & $*$ & $\dagger$ \\
\hline & $\# 42$ & $86(98.85 \%)$ & $1(1.15 \%)$ & $0(0 \%)$ & & \\
\hline & $\# 43$ & $85(97.70 \%)$ & $0(0 \%)$ & $2(2.30 \%)$ & $*$ & \\
\hline & $\# 44$ & $79(90.80 \%)$ & $3(3.45 \%)$ & $5(5.75 \%)$ & $*$ & \\
\hline & $\# 45$ & $84(96.55 \%)$ & $0(0 \%)$ & $3(3.45 \%)$ & $*$ & \\
\hline & $\# 46$ & $87(100 \%)$ & $0(0 \%)$ & $0(0 \%)$ & & \\
\hline & $\# 47$ & $80(91.95 \%)$ & $0(0 \%)$ & $7(8.05 \%)$ & $*$ & \\
\hline & $\# 48$ & $80(91.95)$ & $6(6.90 \%)$ & $1(1.15 \%)$ & $*$ & \\
\hline & $\# 49$ & $82(94.25 \%)$ & $1(1.15 \%)$ & $4(4.60 \%)$ & $*$ & \\
\hline & $\# 50$ & $83(95.40 \%)$ & $0(0 \%)$ & $4(4.60 \%)$ & $*$ & \\
\hline & $\# 51$ & $65(74.71 \%)$ & $3(3.45 \%)$ & $19(21.84 \%)$ & $*$ & $t$ \\
\hline & $\# 52$ & $81(93.10 \%)$ & $0(0 \%)$ & $6(6.90 \%)$ & $*$ & $t$ \\
\hline & $\# 53$ & $81(93.10 \%)$ & $2(2.30 \%)$ & $4(4.60 \%)$ & $*$ & \\
\hline & $\# 54$ & $78(89.66 \%)$ & $0(0 \%)$ & $9(10.34 \%)$ & $*$ & $t$ \\
\hline & $\# 55$ & $86(98.85 \%)$ & $1(1.15 \%)$ & $0(0 \%)$ & & \\
\hline & $\# 56$ & $85(97.70 \%)$ & $0(0 \%)$ & $2(2.30 \%)$ & $*$ & \\
\hline & $\# 57$ & $54(62.07 \%)$ & $6(6.90 \%)$ & $27(31.03 \%)$ & $*$ & $t$ \\
\hline & $\# 58$ & $68(78.16 \%)$ & 16(18.39\%) & $3(3.45 \%)$ & $*$ & \\
\hline & $\# 59$ & $86(98.85 \%)$ & $1(1.15 \%)$ & $0(0 \%)$ & & \\
\hline & $\# 60$ & $68(78.16 \%)$ & $10(11.49 \%)$ & $9(10.34 \%)$ & $*$ & $t$ \\
\hline & $\# 61$ & $86(98.85 \%)$ & $1(1.15 \%)$ & $0(0 \%)$ & & \\
\hline & $\# 62$ & $76(87.36 \%)$ & $6(6.90 \%)$ & $5(5.75 \%)$ & $*$ & \\
\hline
\end{tabular}

Nota. $\left({ }^{*}\right)=$ Indica ítems que fueron considerados para revisión; $(\dagger)$ = indica ítems que fueron modificados. 


\section{Estudio 3, Dephi 2}

Setenta y tres $(\mathrm{N}=73)$ terapeutas ocupacionales participaron en este estudio y trabajaron en los 62 ítems revisados y/o modificados durante el Estudio 2, Delphi 1. Las opciones en este estudio fueron reducidas a "mantener" o "eliminar". El porcentaje de acuerdo para "eliminar" osciló entre el $0 \%$ y $24.6 \%$ y el porcentaje de acuerdo para "mantener" entre $55 \%$ y $100 \%$. De este modo, se mantuvieron los sesenta y dos ítems del CMRS y que constituyen la version final del cuestionario.

\section{Estudio 4, Dephi 3}

Sesenta $(N=60)$ terapeutas ocupacionales completaron este estudio que se centró en clasificar todos los ítems del cuestionario en dos taxonomías: Aspectos de medición en cuatro categorías (hipo-responsividad, hiper-responsividad, social y planificación motriz) y pertenencia a una de seis escalas sensoriales (auditiva, gusto/olfato, propiocepción, táctil, vestibular o visual). Las opciones fueron forzadas en dos revisiones. Primero, los participantes clasificaron cada ítem como perteneciente a un modo de expresión en una de cuatro categorías. Segundo, los mismos ítems fueron repetidos y los participantes los clasificaron como pertenecientes a una de seis escalas sensoriales. Algunos ítem fueron clasificados en dos o más categorías y/o escalas como se indica en la Tabla 3. Cada clasificación se revisó en relación a la información obtenida en la revisión sistemática de la literatura para su asignación final en las categorías y escalas establecidas. Un ítem fue clasificado en una escala adicional "dolor" por su importancia en el contexto de casos que podrían expresar riesgo de daño o lesión. La tabla 3 indica la distribución de los ítems del CMRS en sus respectivas categorías y escalas. La tabla 4 ilustra los 62 ítems en sus respectivas categorías y escalas luego ser revisados en relación a la revisión sistemática de la literatura.

Tabla 3

Clasificación de los ítems de CMRS en sus diferentes categorías y escalas

\begin{tabular}{|c|c|c|c|c|c|c|}
\hline \multirow{2}{*}{ Item } & Hipo-Responsividad* & Hiper-Responsividad* & $\begin{array}{c}\text { Item } \\
\text { Mixtos }\end{array}$ & Social & $\begin{array}{c}\text { Planificación } \\
\text { Motriz }\end{array}$ & $\begin{array}{c}\text { Escala } \\
\text { Mixta**+† }\end{array}$ \\
\cline { 2 - 7 } & 29 & $26+$ & $24 *+$ & 10 & 17 & 14 \\
\hline
\end{tabular}

Nota. $(*)$ Items clasificados únicamente en esta área; $(*+)$ Items clasificados como expresión de hipo/hiper-responsividad; $(* *+\dagger)$ Items clasificados en dos escalas (ej. propioceptivo-vestibular)

Tabla 4

Items finales del CMRS clasificados en cuatro categorías de expresión y seis escalas sensoriales

\begin{tabular}{|c|c|c|c|c|c|}
\hline Item & $\mathrm{H}-$ & $\mathrm{H}+$ & $\mathrm{S}$ & PM & Escala \\
\hline $\begin{array}{l}\text { \#1. Parece ser sensible a la luz (ej. cierra los ojos o baja la mirada al salir al sol } \\
\text { o en lugares con luz artificial) }\end{array}$ & & * & & & VIS \\
\hline \#2. Se acerca demasiado al papel o superficie cuando dibuja, copia o pinta & * & & & & VIS \\
\hline \#3. Mueve sus manos/dedos cerca de sus ojos & * & & & & VIS \\
\hline \#4. Coloca sus ojos hacia los lados (mira de costado o de reojo) & & $*$ & & & VIS \\
\hline $\begin{array}{l}\text { \#5. Tiene dificultad con actividades visuales para su edad (ej. rompecabezas, } \\
\text { construir con bloques, copiar formas) }\end{array}$ & * & & & * & VIS \\
\hline \#6. Parece no notar algunos objetos en el ambiente (ej. se tropieza con objetos) & * & & & $*$ & VIS \\
\hline \#7. Presta excesiva atención a ciertas imágenes, letras, números o símbolos & * & * & & $*$ & VIS \\
\hline \#8. Pestañea o se frota los ojos con frecuencia & & * & & & VIS \\
\hline $\begin{array}{l}\text { \#9. Se distrae con información visual (ej. necesita estar en un ambiente libre } \\
\text { de distracciones para poner atención o aprender algo nuevo) }\end{array}$ & & * & & & VIS \\
\hline
\end{tabular}




\begin{tabular}{|c|c|c|c|c|c|}
\hline Item & $\mathrm{H}-$ & $\mathrm{H}+$ & $\mathrm{S}$ & PM & Escala \\
\hline \#10. Inclina su cabeza cuando mira a algunos objetos, imágenes o personas & * & & & & $\begin{array}{l}\text { VIS- } \\
\text { VEST }\end{array}$ \\
\hline$\# 11$. Prefiere comidas de colores específicos & & * & & & VIS \\
\hline \#12. Muestra reacciones exageradas a algunos sabores o aromas & & * & & & $\mathrm{G} / \mathrm{O}$ \\
\hline $\begin{array}{l}\text { \#13. Algunas comidas, olores o sabores le producen arcadas y/o ganas de } \\
\text { vomitar }\end{array}$ & & * & & & $\mathrm{G} / \mathrm{O}$ \\
\hline \#14. Tiene preferencias muy marcadas por sabores particulars & * & * & & & $\mathrm{G} / \mathrm{O}$ \\
\hline $\begin{array}{l}\text { \#15. Tiende a oler objetos, materiales y texturas mucho más que otros niños } \\
\text { de su edad }\end{array}$ & * & & & & $\mathrm{G} / \mathrm{O}$ \\
\hline $\begin{array}{l}\text { \#16. Come o mastica objetos no comestibles (ej. tierra, pintura, plastilina, lá- } \\
\text { piz, cuello de la ropa) }\end{array}$ & $*$ & & & & $\begin{array}{l}\text { G/O- } \\
\text { PROP }\end{array}$ \\
\hline \#17. Le desagrada el olor de los perfumes, desodorantes o artículos de limpieza & & * & & & $\mathrm{G} / \mathrm{O}$ \\
\hline$\# 18$. El aroma o gusto de las comidas limita su dieta significativamente & & * & & & $\mathrm{G} / \mathrm{O}$ \\
\hline \#19. Tiene una dieta limitada & & * & & & $\begin{array}{l}\text { G/O- } \\
\text { TAC }\end{array}$ \\
\hline$\# 20$. Se da cuenta de la presencia de olores fuertes en el ambiente & & $*$ & & & $\mathrm{G} / \mathrm{O}$ \\
\hline $\begin{array}{l}\text { \#21. Llora o se molesta con los sonidos de artículos del hogar (ej. aspiradora, } \\
\text { secador de pelo, radio, timbre, batidora) }\end{array}$ & & * & & & AUD \\
\hline$\# 22$. El volumen de su voz es muy alto o muy bajo & $*$ & * & $*$ & & AUD \\
\hline \#23. Responde inmediatamente a su nombre & & & $*$ & & AUD \\
\hline $\begin{array}{l}\# 24 \text {. Se confunde cuando se le pide algo verbalmente (ej. tiene dificultades } \\
\text { siguiendo instrucciones verbales) }\end{array}$ & * & & * & $*$ & AUD \\
\hline $\begin{array}{l}\text { \#25. Se cubre los oídos en presencia de sonidos que no molestan a otras per- } \\
\text { sonas (ej. campana, sirena, silbato, timbre, artículos electrodomésticos) }\end{array}$ & & * & & & AUD \\
\hline \#26. Repite frases, palabras, o sonidos & $*$ & & * & & AUD \\
\hline $\begin{array}{l}\text { \#27. Intenta controlar el volumen de diferentes sonidos (radio, } \\
\text { televisión, vOZ, música) }\end{array}$ & & $*$ & & & AUD \\
\hline $\begin{array}{l}\text { \#28. Evita y se desorganiza en ambientes con muchos estímulos (shopping, } \\
\text { supermercado, tienda, transporte público, fiestas de cumpleaños, restaurant) }\end{array}$ & & * & $*$ & & AUD \\
\hline $\begin{array}{l}\# 29 \text {. Se distrae en presencia de sonidos de fondo (radio, televisión, } \\
\text { conversación, autos) }\end{array}$ & & $*$ & & & AUD \\
\hline $\begin{array}{l}\text { \#30. Hace mucho ruido con objetos, juguetes o materiales } \\
\text { (e.j. los golpea, murmura) }\end{array}$ & * & & & & AUD \\
\hline $\begin{array}{l}\text { \#31. Llora o parece sentir naúseas luego de viajes en el auto } \\
\text { o transporte public }\end{array}$ & & * & & & VEST \\
\hline $\begin{array}{l}\text { \#32. Se resiste a utilizar equipos con movimiento (ej. ascensor, escalera } \\
\text { mecánica, calesita, parque de diversiones, sube y baja) }\end{array}$ & & * & & & VEST \\
\hline $\begin{array}{l}\text { \#33. Le agradan las actividades que incluyen movimientos intensos (e.j. ser } \\
\text { lanzado en el aire, parques de diversiones, trampolín, correr) }\end{array}$ & * & & & & VEST \\
\hline $\begin{array}{l}\text { \#34. Busca apoyarse cuando está en la mesa (e.j. sostiene la cabeza con sus } \\
\text { manos, se apoya en otras personas) }\end{array}$ & * & & & & $\begin{array}{l}\text { PROP- } \\
\text { VEST }\end{array}$ \\
\hline $\begin{array}{c}\text { \#35. Le desagradan los cambios de posición (ej. moverse en la silla, saltar, } \\
\text { subir o bajar escaleras, treparse, hamacarse) }\end{array}$ & & * & & & VEST \\
\hline $\begin{array}{l}\text { \#36. Parece ansioso/a en el parque y durante actividades que requieren saltar, } \\
\text { girarse, treparse, bajarse o subirse }\end{array}$ & & * & & * & VEST \\
\hline \#37. Se cae con frecuencia & * & & & * & $\begin{array}{l}\text { PROP- } \\
\text { VEST }\end{array}$ \\
\hline
\end{tabular}




\begin{tabular}{|c|c|c|c|c|c|}
\hline Item & $\mathrm{H}-$ & $\mathrm{H}+$ & $\mathrm{S}$ & PM & Escala \\
\hline $\begin{array}{l}\text { \#38. Tiene mucha más energía que otros niños de su edad cuando se despi- } \\
\text { erta (ej. salta en la cama, corre, se mueve excesivamente, es muy activo) }\end{array}$ & * & & & $*$ & $\begin{array}{l}\text { PROP- } \\
\text { VEST }\end{array}$ \\
\hline $\begin{array}{l}\text { \#39. Tiene dificultad con actividades que requieren equilibrio (triciclo, bicicle- } \\
\text { ta, juegos de equilibrio o balancearse) }\end{array}$ & $*$ & & & $*$ & $\begin{array}{l}\text { VEST- } \\
\text { PROP }\end{array}$ \\
\hline \#40. Le es difícil atrapar pelotas cuando se compara con niños de su edad & * & & * & * & $\begin{array}{l}\text { PROP- } \\
\text { VEST }\end{array}$ \\
\hline $\begin{array}{l}\text { \#41. Le molesta jugar con pintura de dedos, crema de afeitar u otros materia- } \\
\text { les blando, pegajosos o granulosos }\end{array}$ & & * & & & TAC \\
\hline \#42. Evita alimentos blandos como pure, yogurt o flan & $*$ & $*$ & & & TAC \\
\hline \#43. Evita alimentos que requieren masticar (ej. carne) & & * & & * & $\begin{array}{l}\text { PROP- } \\
\text { TAC }\end{array}$ \\
\hline \#44. Frota sus manos, cara, cuerpo en superficies, texturas u objetos & $*$ & & & & TAC \\
\hline \#45. Evita el contacto físico con algunas superficies, objetos o texturas & & $*$ & & & TAC \\
\hline \#46. Toca objetos y superficies con su lengua, labios, boca, o cara & $*$ & & & & TAC \\
\hline $\begin{array}{l}\text { \#47. Presenta estrés durante actividades de higiene o arreglo personal (ej. ce- } \\
\text { pillado de dientes, lavado de cara, cortado de uñas, lavado de cabello, baño, } \\
\text { cortado de cabello) }\end{array}$ & & * & & & TAC \\
\hline \#48. Lleva objetos no comestibles a la boca & * & & & & $\begin{array}{l}\text { TAC- } \\
\text { PROP }\end{array}$ \\
\hline \#49. Se niega a utilizar algunas prendas de vestir (ej. sweaters de lana, nylon, etc.) & & $*$ & & & TAC \\
\hline $\begin{array}{l}\text { \#50. Tiene dificultad con el espacio personal (ej. toca o se acerca demasiado } \\
\text { a otros niños o personas) }\end{array}$ & $*$ & & * & $*$ & $\begin{array}{l}\text { TAC- } \\
\text { PROP }\end{array}$ \\
\hline \#51. Parece no sentir el dolor de la misma forma que otros niños de su edad & $*$ & & & & $\mathrm{TAC} / \mathrm{D}$ \\
\hline $\begin{array}{l}\text { \#52. Le desagrada el contacto o proximidad con otras personas (e.j. durante el } \\
\text { juego en el salón de clase, formando una fila, en el parque) }\end{array}$ & & $*$ & * & & TAC \\
\hline \#53. Rompe juguetes, objetos y materiales con facilidad & $*$ & & & $*$ & PROP \\
\hline $\begin{array}{l}\text { \#54. Tiene dificultades para medir su fuerza o utiliza demasiada o muy poca } \\
\text { fuerza con algunos objetos (e.j. lápiz, crayón, tenedor, cuchara, cerrar la puerta) }\end{array}$ & * & & & * & PROP \\
\hline $\begin{array}{l}\text { \#55. Busca actividades como moverse, correr, saltar, treparse, lanzarse, col- } \\
\text { garse, patear más que otros niños de su edad }\end{array}$ & * & & & & $\begin{array}{l}\text { PROP- } \\
\text { VEST }\end{array}$ \\
\hline \#56. Sus músculos parecen blandos y débiles & $*$ & & & & PROP \\
\hline \#57. Usa movimientos muy amplios y bruscos cuando no se requieren & $*$ & & & $*$ & PROP \\
\hline $\begin{array}{l}\text { \#58. Mastica objetos (e.j. cuello de su camisa, lápiz, crayón, puño de su man- } \\
\text { ga, goma de borrar) }\end{array}$ & * & & & & PROP \\
\hline $\begin{array}{l}\text { \#59. Es brusco en sus interacciones con otros niños (ej. golpea, empuja, aprie- } \\
\text { ta, pellizca, muerde) }\end{array}$ & * & * & * & * & $\begin{array}{l}\text { PROP- } \\
\text { TACT }\end{array}$ \\
\hline \#60. Es torpe en comparación a niños de su edad & $*$ & & & $*$ & PROP \\
\hline \#61. Camina en puntas de pie & * & * & & & $\begin{array}{l}\text { PROP- } \\
\text { TAC }\end{array}$ \\
\hline $\begin{array}{l}\text { \#62. Se tropieza y choca con objetos en el ambiente (ej. personas, muebles, } \\
\text { marcos de la puerta) }\end{array}$ & * & & * & $*$ & PROP \\
\hline
\end{tabular}

Nota. $\mathrm{H}-$ = Hipo-responsividad; $\mathrm{H}+=$ Hiper-responsividad; $\mathrm{S}=$ Social; VIS = Escala Visual; G/O = Escala Gusto/ Olfato; $\mathrm{AU}=$ Escala Auditiva; $\mathrm{VES}=$ Escala Vestibular; $\mathrm{TAC}=$ Escala Táctil; $\mathrm{PROP}=$ Escala Proprioceptiva; $\mathrm{PM}=$ Planificación Motriz; D = Dolor. 


\section{Discusión}

La evaluación de la responsividad sensorial en niños con diagnóstico de autismo es compleja. Para su realización efectiva se require la utilización de instrumentos de medición consensuados formal y sistemáticamente en investigación en el contexto geográfico y socio-cultural en el que se utilizan.

Los resultados preliminares de esta investigación sugieren que en CMRS y sus sesenta y dos ítems revisados prospectivamente por 87, 73 y 60 Terapeutas Ocupacionales podrían explorar el constructo de responsividad sensorial en niños con autismo en Argentina. La factibilidad para utilización en otras regiones podría explorarse en estudios adicionales. Por ejemplo, una versión preliminar de este instrumento modificó 14 ítems y reportó una diferencia estadísticamente significativa entre niños con diagnóstico de autismo y niños típicos en Venezuela (Alvarez, Da Silva y De Pinto, 2015).

Una clasificación taxonómica que utiliza seis escalas sensoriales, 1 ítem adicional de dolor, y 4 categorías que incluyen hipo-responsividad, hiper-responsividad, aspectos sociales y de planificación motriz podrían esclarecer la naturaleza y tipo de impacto en diferentes contextos y ocupaciones cuando se evalúan estos niños. La utilización de muestras de niños típicos podrían establecer rangos de puntajes esperados, parámetros de frecuencia e intensidad en la presencia de ítems o grupos de ítems específicos. Niños con diagnóstico de autismo de diferentes edades, sexo y co-ocurrencia de otros diagnósticos podrían esclarecer estructuras de grupo y factores. Estudios futuros prodrían establecer las propiedades psicométricas como la estabilidad del instrumento en situaciones de test y re-test o la consistencia interna de diferentes escalas. Adicionalmente, la comparación con instrumentos con propiedades psicométricas establecidas prodría asistir en su validez de predicción o concurrente.

Una pequeña proporción de ítems fueron clasificados en más de una categoría (ej. hipo e hiper-responsividad) o escala (ej. propioceptivo-vestibular). Este fenómeno ha sido reportado en revisiones sistemáticas de la literatura y fuentes adicionales (Blanche \& Reinoso, 2007; Greenspan \& Wieder 1997; Hirstein, Iversen \& Ramachandran, 2001) y podría reflejar un modo diferencial de funcionamiento ante la presencia de diferentes aspectos del contexto (Baranek, 2002; Baranek et al., 2013; Little et al., 2011) u otras situaciones. Estos ítems deberían interpretarse con precaución y razonamiento clínico avanzado en el contexto clínico y de investigación.

\section{CONCLUSIÓN}

Los niños con diagnóstico de autismo presentan síntomas sensoriales como parte de su diagnóstico. Estas problemas pueden afectar acciones, tareas, actividades y ocupaciones así como también el desarrollo de hábitos y rutinas saludables y la participación en diferentes contextos. Un método de exámen de esta dimensión incluye la utilización de cuestionarios para los padres o personas que interactúan frecuentemente con estos niños.

La importancia de este estudio reside en el desarroIlo y presentación del Cuestionario para los padres para la Medición de la Responsividad Sensorial en niños con Autismo (CMRS) que fue desarrollado en Argentina mediante una revisión sistemática de la literatura y 3 estudios que utilizaron una metodología Delphi para consensuar la inclusión, exclusión y modificación de items. Todos los ítems fueron revisados prospectivamente por 87, 73 y 60 Terapeutas Ocupacionales con certificación en Integración Sensorial en Argentina. El CMRS consta de 62 ítems en 4 categorías (hipo-responsividad, hiperresponsividad, social y planificación motriz) y 6 escalas sensoriales (visual, auditiva, gusto/olfato, propioceptiva, vestibular y táctil). Estudios futuros podrían esclarecer aspectos adicionales de sus propiedades psicométricas y utilidad en la práctica clínica e investigación.

\section{REFERENCIAS BIBLIOGRÁFICAS}

Al Abdulmohsen, T., \& Kruger, T. C. (2011). The contribution of muscular and auditory pathologies to the symptomatology of autism. Medical Hypotheses, 77(6), 1038-1047. doi:doi:10.1016/j/ mehy.2011.08.044

Alcántara, J., Weisblatt, E., Moore, B., \& Bolton, P. (2004). Speech-innoise perception in high-functioning individuals with autism or Asperger's syndrome. Journal Of Child Psychology \& Psychiatry,45(6), 1107-1114. doi:doi:10.1111/j.1469-7610.2004.t01100303.x 
Alvarez, N., Da Silva, G., \& De Pinto, G. (2015). Comparación del Procesamiento Sensorial de Niños con y sin Diagnóstico de Autismo entre las Edades Comprendidas de 5 a 12 años en Venezuela (Manuscrito No Publicado). Universidad Central de Venezuel, Facultad de Medicina, Escuela de Salud Pública, Terapia Ocupacional. Caracas, Venezuela.

American Psychiatric Association (2013). Diagnostic and statistical manual of mental disorders (5th ed.). Washington, DC: Author.

Alcántara, J., Weisblatt, E., Moore, B., \& Bolton, P. (2004). Speech-innoise perception in high-functioning individuals with autism or Asperger's syndrome. Journal Of Child Psychology \& Psychiatry, 45(6), 1107-1114. doi:doi:10.1111/j.1469-7610.2004.t01-100303.x

Andrews, D., Nonnecke, B., \& Preece, J. (2003). Electronic Survey Methodology: A Case Study in Reaching Hard-to-Involve Internet Users. International Journal of Human-Computer Interaction, 16(2), 185-210. doi:DOI:10.1207/S15327590IJHC1602_04

Ashburner, J., Bennet, L., Rodger, S., \& Ziviani, J. (2013). Understanding the sensory experiences of young people with autism spectrum disorder: A preliminary investigation. Australian Occupational Therapy Journal, 60(3), 171-180. doi:doi:10.1111/1440-1630.12025

Ashburner, J., Bennett, L., Rodger, S., \& Ziviani, J. (2014). Understanding the sensory experiences of young people with autism spectrum disorder: A preliminary investigation. Australian Occupational Therapy Journal, 60(3), 171-180. doi:doi:10.1111/1440-1630.12025

Ausderau, K. K., Furlong, M., Sideris, J., Bulluck, J., Little, L. M., Watson, L. R., . . Baranek, G. T. (2014). Sensory subtypes in children with autism spectrum disorder: latent profile transition analysis using a national survey of sensory features. J Child Psychol Psychiatry, 55(8), 935-944. doi:10.1111/jcpp.12219

Ayres. (2005). Sensory integration and the child (25th anniversary edition ed.). Los Angeles, CA: Western Psychological Services.

Baker, A. E., Lane, A., Angley, M. T., \& Young, R. L. (2008). The relationship between sensory processing patterns and behavioural responsiveness in autistic disorder: A pilot study. Journal of Autism and Developmental Disorders, 38(5), 867-875. doi:doi:10.1007/ s10803-007-0459-0

Baranek, G. T. (2002). Efficacy of sensory and motor interventions for children with autism. J Autism Dev Disord, 32(5), 397-422.

Baranek, G. T., Watson, L. R., Boyd, B. A., Poe, M. D., David, F. J., \& McGuire, L. (2013). Hyporesponsiveness to social and nonsocial sensory stimuli in children with autism, children with developmental delays, and typically developing children. Development and Psychopathology, 25, 307-320. doi:http://dx.doi.org/10.1017/ S0954579412001071

Baum, S. H., Stevenson, R. A., \& Wallace, M. T. (2015). Testing sensory and multisensory function in children with autism spectrum disorder. J Vis Exp(98), e52677. doi:10.3791/52677
Blanche, E. I., Bodison, S., Chang, M., \& Reinoso, G. (2012). Development of the comprehensive observations of proprioception (COP): validity, reliability, and factor analysis. Am J Occup Ther, 66(6), 691-698. doi:10.5014/ajot.2012.003608

Blanche, E. I., \& Reinoso, G. (2007). Literature Review: Sensory Processing Deficits in Autism Spectrum Disorder. Chilean Journal of Occupational Therapy, 7, 59-68. doi:DOI: 10.5354/07175346.2007.79

Blanche, E. I., \& Reinoso, G. (2008). The use of clinical observations to evaluate proprioceptive and vestibular functions. OT Practice, 13(17).

Blanche, E. I., Reinoso, G., Chang, M. C., \& Bodison, S. (2012). Proprioceptive processing difficulties among children with autism spectrum disorders and developmental disabilities. Am J Occup Ther, 66(5), 621-624. doi:10.5014/ajot.2012.004234

Brooks, K. W. (1979). Delphi technique: Expanding applications. . North Central Association Quaterly, 54(3), 377-385.

Case-Smith, J., Weaver, L. L., \& Fristad, M. A. (2014). A systematic review of sensory processing interventions for children with autism spectrum disorders. Autism, 19(2), 133-148. doi:doi: $10.1177 / 1362361313517762$

Case-Smith, J., Weaver, L. L., \& Fristad, M. A. (2015). A systematic review of sensory processing interventions for children with autism spectrum disorders. Autism, 19(2), 133-148. doi:10.1177/1362361313517762

Cheung, P. P., \& Siu, A. M. (2009). A comparison of patterns of sensory processing in children with and without developmental disabilities. Research in Developmental Disabilities, 30(6), 1468-1480. doi:doi:10.1016/j.ridd.2009.07.009.

Couper, M. P. P. O. Q. (2000). Web-based surveys: A Review of Issues and Approaches. Public Opinion Quaterly, 64, 464-494.

Davis, R. A. O., Bockbrader, M. A., Murphy, R. R., Hetrick, W. P., \& O'Donnell, B. F. (2006). Subjective perceptual distortion and visual dysfunction in children with autism. . Journal of Autism and Developmental Disorders,, 36(2), 199-210. doi:doi:10.1007/s10803-005$0055-0$

Dunn, W., Saiter, J., \& Rinner, L. (2002). Asperger syndrome and sensory processing: A conceptual model and guidance for intervention planning. Focus on Autism \& Other Developmental Disabilities, 17(3), 172. doi:doi:10.1177/10883576020170030701

Fowler, J., \& Floyd, J. (1995). Improving Survey Questions: Design and Evaluation (Vol. 38). Thousand Oaks: Sage Publications.

Greatorex, J. (2000). An accessible analytical approach for investigating what happens between the rounds of a Delphi study. Journal of Advanced Nursing, 32(4), 1016-1024.

Greffou, S., Bertone, A., Hahler, E.-M., Hanssens, J.-M., Mottron, L., \& Faubert, J. (2012). Postural Hypo-Reactivity in Autism is Contingent on Development and Visual Environment: A Fully Immersive Virtual Reality Study. Journal of Autism \& Developmental Disorders, 42(6), 961-970. doi:10.1007/s10803-011-1326-6 
Hasson, F., Keeney, S., \& Mckenna, H. (2000). Research guidelines for the Delphi survey technique. Journal of Advanced Nursing, 32(4), 1008-1015.

Hsu, C., \& Sandord, B. A. (2007). The Delphi technique: Making sense of consensus. Practical Assessment, Research \& Evaluation, 12(10), $1-7$.

James, K., Miller, L. J., Schaaf, R., Nielsen, D. M., \& Schoen, S. A. (2011). Phenotypes within sensory modulation dysfunction. Comprehensive Psychiatry, 52(6), 715-724. doi:http://dx.doi.org/10.1016/j. comppsych.2010.11.010

Kanner, L. (1943). Autistic disturbances of affective contact. Nervous Child, 2, 217-250.

Kern, J. K., Garver, C., Carmody, T., Andrews, A., Trivedy, J. K., \& Mehta, J. A. (2007). Examining sensory modulation in individuals with autism as compared to community controls. Autism, 10(5), 480-494.

Kirby, A. V., Dickie, V. A., \& Baranek, G. T. (2015). Sensory experiences of children with autism spectrum disorder: in their own words. Autism, 19(3), 316-326. doi:10.1177/1362361314520756

Lane, A. E., Dennis, S. J., \& Geraghty, M. E. (2011). Brief report: Further evidence of sensory subtypes in autism. Journal of Autism and Developmental Disorders, 41(6), 826. doi:doi:10.1007/s10803-0101103-y

Little, L. M., Ausderau, K., Sideris, J., \& Baranek, G. T. (2015). Activity Participation and Sensory Features Among Children with Autism Spectrum Disorders. J Autism Dev Disord. doi:10.1007/s10803015-2460-3

Little, L. M., C., F. A., Houser, M. B., Guckian, L., Carbine, K., David, F. J., \& Baranek, G. T. (2011). Psychometric validation of the Sensory Experiences Questionnaire. American Journal of Occupational Therapy, 65(2), 207-210. doi:doi: 10.5014/ajot.2011.000844

Ludlow, A., Mohr, B., Whitmore, A., Garagnani, M., Pulvermüller, F., \& Gutierrez, R. (2014). Auditory processing and sensory behaviours in children with autism spectrum disorders as revealed by mismatch negativity. Brain \& Cognition,, 86, 55-63. doi: doi:10.1016/j. bandc.2014.01.016

O'Donnell, S., Deitz, J., Kartin, D., Nalty, T., \& Dawson, G. (2012). Sensory processing, problem behavior, adaptive behavior, and cognition in preschool children with autism spectrum disorders. American Journal of Occupational Therapy, 66(5), 586-594. doi:doi:10.5014/ajot.2012.004168

Okoli, C., \& Pawlowski, S. D. (2004). The Delphi method as a research tool: An example,design considerations and applications. Information \& Management, 42(1), 15-29. doi:doi:10.1016/j.im.2003.11.002

Paterson, H., \& Peck, K. (2011). Sensory processing ability and eating behaviour in children with autism. Journal of Human Nutrition and Dietetics, 24(4), 301. doi:doi:10.1111/j.1365-277X.2011.01175_31.x
Pill, J. (1971). The Delphi method: Substance, context, and critique and an annotated bibliography. Socio-Economic Planning Science, 5(57-71).

Stein, L. I., Jane, C. J., Williams, M. E., Dawson, M. E., Polido, J. C., \& Cermak, S. (2014). Physiological and behavioral stress and anxiety in children with autism spectrum disorders during routine oral care. Biomedical Research International, 2014. doi:http://dx.doi. org/10.1155/2014/694876

Talay-Ongan, A., \& Wood, K. (2000). Unusual sensory sensitivities in autism: A possible crossroads. International Journal of Disability, Development and Education, 47(2), 201-212. doi:doi:10.1080/713671112

Tomchek, S. D., \& Dunn, W. (2007). Sensory processing in children with and without autism: A camparative study using the short sensory profile. The American Journal of Occupational Therapy, 61(2), 190200. doi:doi:10.5014/ajot.61.2.190

Tomchek, S. D., Huebner, R. A., \& Dunn, W. (2014). Patterns of sensory processing in children with an autism spectrum disorder. . Research in Autism Spectrum Disorders,, 8(9), 1214-1224. doi:doi:10.1016/j. rasd.2014.06.006

Weible, R., \& Wallace, J. (1999). The impact of the Internet on data collection. Marketing Research, 10(3), 19-23.

Wigham, S., Rodgers, J., South, M., McConachie, H., \& Freeston, M. (2015). The interplay between sensory processing abnormalities, intolerance of uncertainty, anxiety and restricted and repetitive behaviours in autism spectrum disorder. . Journal of Autism and Developmental Disorders,, 45(4), 943-952. doi:doi:10.1007/s10803014-2248-x

Tomchek, S. D., Huebner, R. A., \& Dunn, W. (2014). Patterns of sensory processing in children with an autism spectrum disorder. Research in Autism Spectrum Disorders,8(9), 1214-1224. doi:doi:10.1016/j. rasd.2014.06.006

Thorpe, H., \& Crispin, J. n. d. (n.d). Sensory processing resource pack: Early years: Working with children with sensory processing differences in early years settings. Retrieved from http://www.leics.gov. uk/early_years_sensory_processing_resource_pack_multiagency_final_march_2014.pdf

Weible, R., \& Wallace, J. (1999). The impact of the Internet on data collection. Marketing Research, 10(3), 19-23.

Weimer, A. K., Schatz, A. M., Lincoln, A., Ballantyne, A., \& Trauner, D. A. (2001). "Motor" impairment in Asperger syndrome: Evidence for a deficit in proprioception. Journal of Developmental and Behavioral Pediatrics, 22(2), 92-101. doi:d oi:10.1097/00004703-200104000-00002

Wigham, S., Rodgers, J., South, M., McConachie, H., \& Freeston, M. (2015). The interplay between sensory processing abnormalities, intolerance of uncertainty, anxiety and restricted and repetitive be- 
haviours in autism spectrum disorder. . Journal of Autism and Developmental Disorders,, 45(4), 943-952. doi:doi:10.1007/s10803014-2248-x

Yonkman, J., Lawler, B., Talty, J., O’Neil, J., \& Bull, M. (2013). Safely transporting children with autism spectrum disorder: Evaluation and intervention. American Journal of Occupational Therapy, 67(6), 711-716. doi:American Journal of Occupational Therapy

Zwaigenbaum, L., Bryson, S., Rogers, T., Roberts, W., Brian, J., \& Szatmari, P. (2005). Behavioral manifestations of autism in the first year of life. The International Society For Developmental Neuroscience, 23(2-3), 143-152. doi:doi:10.1016/j.ijdevneu.2004.05.001 